\title{
An Object-Oriented Model of Measurement Systems
}

\author{
Qingping Yang, Member, IEEE, and Clive Butler
}

\begin{abstract}
This paper presents a general object-oriented model for measurement systems. The limitations of the conventional function-oriented models are examined in the light of the generalized concept of measurement and its theoretical framework proposed previously by the authors. The proposed model identifies five classes of objects, i.e., measured object, measuring instrument, reference standard, human observer, and operating environment. Each is characterized by its own attributes and operations or functions at three levels, i.e., internal, operational, and environmental. The interactions between them are also modeled, including the coupling between the measured object and the measuring instrument, the human-instrument interface, the calibration, and the interference. It serves as both a modeling framework and a practical tool for description, analysis and design, and, in particular, for computer-aided analysis and design of a measuring system. It will find applications in instrumentation engineering and measurement research and education.
\end{abstract}

Index Terms-Measurement science, measurement system data handling, modeling, object-oriented methods, system analysis and design, virtual instrumentation.

\section{INTRODUCTION}

$\mathbf{M}$ EASUREMENT systems may be modeled using various representation schemes at different levels of abstraction, e.g., linguistic, graphical, and mathematical. As an abstraction of some measurement system, a model only captures the essential system characteristics, with irrelevant details omitted to reduce the system complexity. A welldeveloped model does not only function as a useful description of a measurement system, it also greatly facilitates its analysis and design. Modeling has played an increasing role in instrumentation and measurement systems, especially in complicated measurement systems and, more recently, virtual instrumentation.

A model is inevitably limited by the explicit and/or implicit assumptions made in its design and use. While the explicit assumptions are usually justified to reduce the system complexity, the implicit assumptions often reflect an underlying conceptual framework and originate from a priori propositions about measurement systems. Practical difficulties will often arise if its underlying theoretical framework is inadequate or flawed.

The underlying conceptual framework has been addressed in [1], in the epistemological context of measurement. A generalized concept of measurement based upon a "knowledge pyramid" was also proposed in [1], i.e., measurement transforms the measured properties or attributes through internal

Manuscript received June 1, 1997; revised May 1, 1998.

The authors are with The Brunel Centre for Manufacturing Metrology, Brunel University, Middlesex, UB8 3PH, U.K. (e-mail: emstqqy@brunel.ac.uk).

Publisher Item Identifier S 0018-9456(98)06311-6.

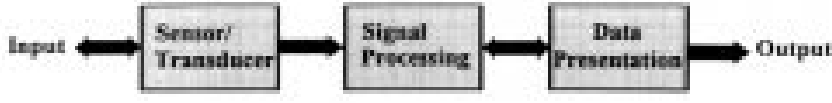

Fig. 1. Functional representation of a measurement system.

symbolic representation directly into knowledge: measured properties $\Rightarrow$ symbolic data $\Rightarrow$ knowledge, where the acquired knowledge may be low-level facts (e.g., the magnitudes of measurands), or in a structural or relational form (e.g., empirical models). The former is essentially the conventional result of measurement.

This paper will discuss the modeling of measurement systems, in particular, an object-oriented model of measurement systems. But the discussions will be made in the light of the generalized knowledge-driven concept of measurement and its conceptual framework [1].

\section{Conventional Models And Their Limitations}

Traditionally, measurement systems are usually modeled with functional representations. The architecture of these representations typically consists of three basic stages, the input stage including a sensor or transducer, the signal processing stage, and the output stage, each of them represented as input-output functional blocks or elements, as shown in Fig. 1.

Functional representations are well established. This kind of model is often used as the basis for the classification of measuring instruments. They are very useful for studying static and dynamic characteristics through transfer functions of each element. They can also be computer-based models, using conventional functional programming languages.

Common to the modeling in various problem domains, the intrinsic limitations of a functional model arise from its low level of abstraction. Its reusuability is very poor as it is intended for specific systems. Despite of the wide use of structural or modular design concept, it is rather inflexible and often difficult to modify. The size of this kind of model, especially a computer-based one, often increases exponentially with complexity.

In the modeling of measurement systems, functional models tend to be oversimplified, neglecting some important aspects of a measurement system, e.g., the coupling interactions between the instrument and the measured object, although work has recently been reported to extend these models [2]-[3]. As originated mainly from control engineering, functional models of measurement systems have often failed to address some issues which are of theoretical and practical importance to measurement, e.g., operating space of measurement [4]. As a result, incomplete descriptions of measuring systems are 
widely used, and the distinctiveness of measurement science has been seriously obscured. This is probably one of the most important reasons why measurement science has attracted little theoretical interests, compared with control engineering.

\section{OBject-ORIENTED Model of A Measurement System}

\section{A. Object-Oriented Methods}

The object-oriented approach views a system as a collection of discrete objects that contain both data structure and behavior. The data structures and behavior or operations of an object are defined by a class. An object is an instance of a class. Objects can interact with other objects.

The history of object-oriented methods is closely associated with computer programming. The use of object-orientation first appeared in the development of a discrete event simulation language (Simula) in Norway 30 years ago [5]. As they began to mature in the late 1980's, object-oriented methods shifted to other areas, e.g., system analysis and design. Indeed, they have become a general modeling approach in the past ten years [6].

The power of the object-oriented approach lies largely in its closeness to the natural view of the real-world. By raising the level of abstraction from the function-level to the object-level, it focuses on the real-world aspects of a system and provides a better model of the problem space. Its consistency with the real-world also provides a unifying platform for all the related activities, e.g., analysis, design, and implementation.

The essential characteristics of the object-oriented approach include information hiding or encapsulation, inheritance, and piecewise refinement. Objects encapsulate both their data structures and operations and they thus work at a higher level of abstraction. This makes the object-oriented model more stable than the convectional functional model because the changes in operations or functions are decoupled and are localized within objects.

The inheritance comes from the class hierarchy structure, in which a class can have its own data structure and operations, and can also inherit them from its parent class(es). Although the concept originated in artificial intelligence, its inherent existence in class hierarchy structure is unique with the objectoriented approach. The reusability of similar objects is one of the most important benefits of object-oriented methods.

The feature of piecewise refinement results from the objectorientation and, in particular, its separated external and internal views of an object. Together with encapsulation, it provides a powerful mechanism to achieve high productivity, good maintainability, system integrity, and reliability.

\section{B. Object-Oriented Model of a Measurement System}

Although there is much literature regarding object-oriented methods, it is nontrivial to develop a sound object-oriented model for measurement systems. This is partly because the object-oriented methods have been strongly influenced by computer programming, which has often obscured the nature of the approach, and it is partly also because of lacking a sound conceptual framework in measurement science, as discussed above. The authors have developed an object-oriented model

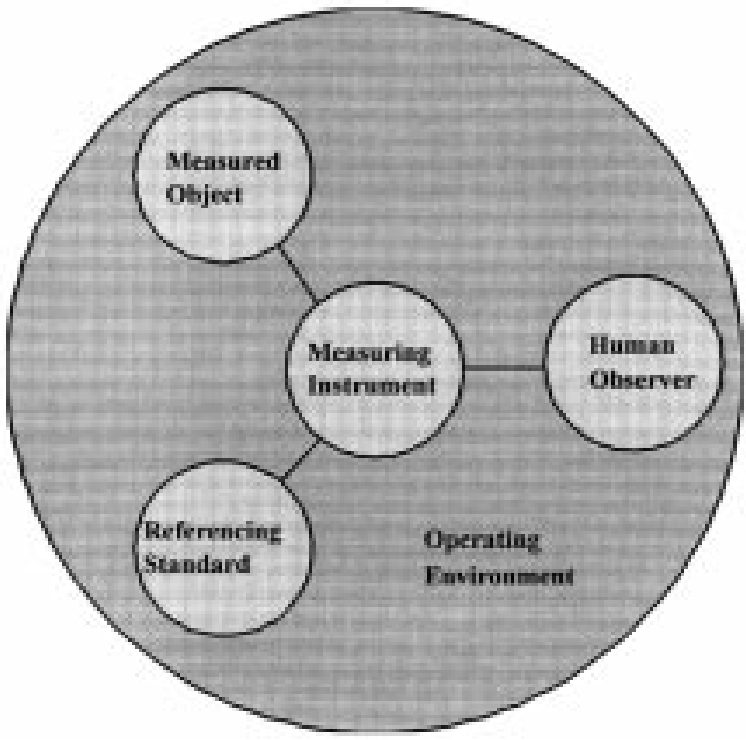

Fig. 2. Architecture of an object-oriented model for measurement systems.

for measurement systems, which may become a practical tool to solve the problems with the existing function-oriented models.

Shown in Fig. 2 is the architecture of an object-oriented model for measurement systems. It captures the important relations in a measurement system and embraces five objects or subsystems, i.e., measured object, measuring instrument, human observer, referencing standard, and operating environment. The model represents a measurement system at three levels internal (measuring instrument), operational and environmental. The measurement can be affected by all the five subsystems and their interactions, with the measuring instrument as the core of the model. The model allows for the systematic study of the objects or subsystems in a measuring system and their interrelations.

Although the model architecture has revealed important aspects of a measurement system, it is necessary to refine it in more detail. The object model shown in Fig. 3 gives further details about the relations between the five classes of objects in a measurement system. Notations used here are based on Rumbaught et al. [6]. A class is represented by a box with three regions, the top region is the class name, the middle one represents the list of attributes, the bottom one the list of operations. For simplicity the lists of attributes and operations are not given in Fig. 3 (some examples given in Figs. 4-6). The associations between object classes are modeled using multiplicity symbols. A solid ball is for "many," meaning zero or more; a hollow ball for "optional," meaning zero or one; a line without multiplicity symbols represents a one-to-one association unless otherwise specified (e.g., 1+).

Due to their importance, four associations have been modeled as four classes in Fig. 3, namely, the coupling between the measured object and the measuring instrument, the human-instrument interface, the interface and the calibration. An association class is represented as a box attached to the association by a loop. 


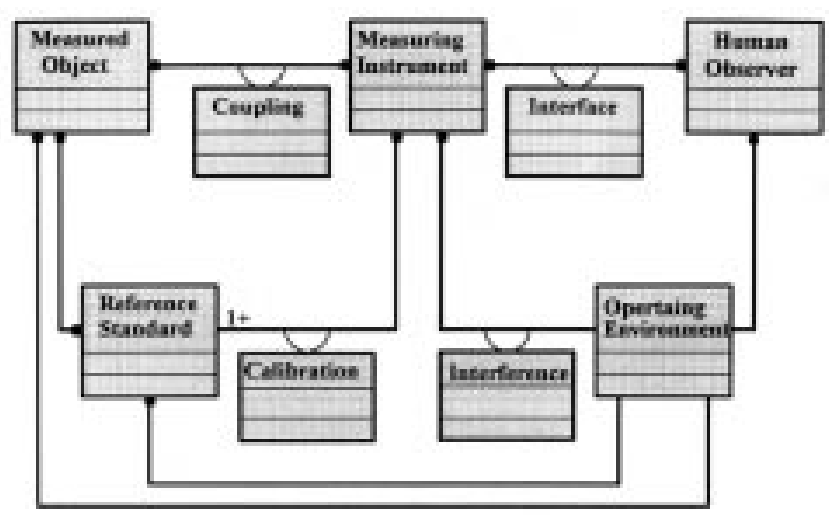

Fig. 3. Object model of a measurement system.

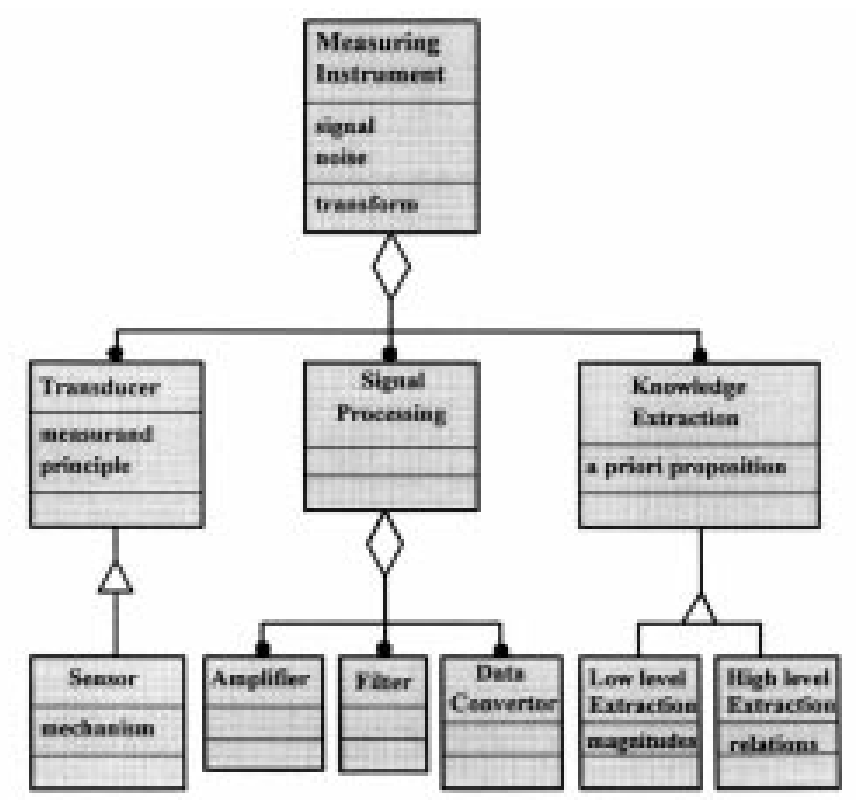

Fig. 4. Object model of a measuring instrument.

It is clear from the model that the operating space of the measurement system is not only determined by measuring instrument, but also by the object instances of the four association classes. It has highlighted problems such as the validity and boundary conditions of a measurement [4].

Of course, each object in the model can be further broken down to more detailed objects according to piecewise refinement. For example, the measuring instrument object is further modeled in Fig. 4, in which a small diamond is drawn at the assembly end of an aggregation relationship. A triangle is used for specialization or inheritance relationship, with the parent class connected to the apex of the triangle and the child class to the base of the triangle. The knowledge extraction class is introduced in accordance with the generalized concept of measurement.

The coupling between the measured object and the measuring instrument has often been neglected in traditional models and may be modeled as shown in Fig. 5. Although looking familiar, loading and sampling, the latter in particular, have profound implications in a measurement system. All measurands go through a natural sampling, both spatially and

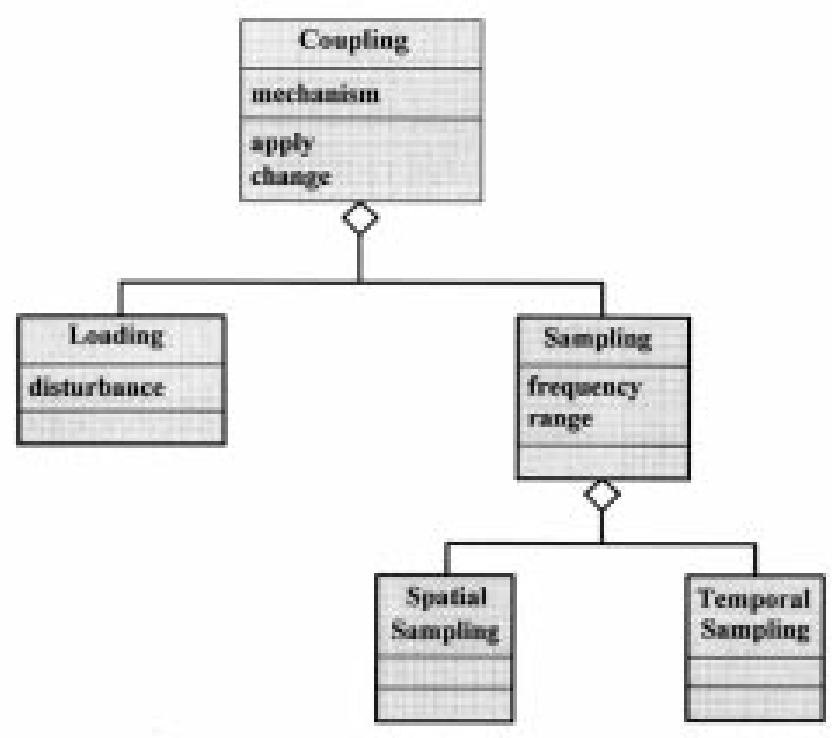

Fig. 5. Object model of the coupling between measured object and measuring instrument.

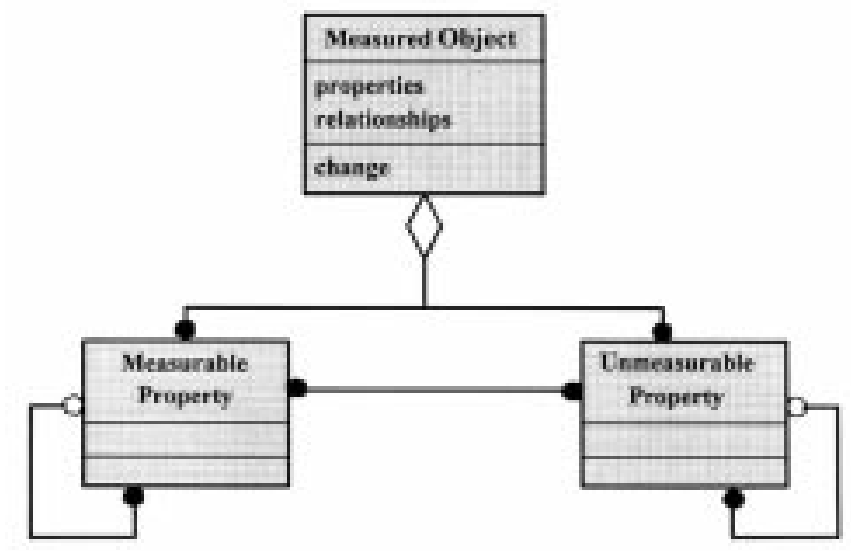

Fig. 6. Object model of the measured object.

temporally. This indicates that many practical measurement problems are inadequately defined and specified. This is particularly true if the measured object is modeled according to the generalized concept of measurement, as shown in Fig. 6, where the relational knowledge about the measured object is usually the purpose of a measurement.

Other classes in Fig. 3 can be modeled using similar techniques. Together with Fig. 3, they represent the object model of a measurement system. An object-oriented model also deals with functional and dynamic aspects of a measurement system [6]. They are compatible with the conventional concept of measurement, and an object-oriented model has thus accommodated conventional functional models.

\section{Advantages and Limitations of the Proposed Model}

As an instance of an object-oriented model class, the proposed model will inherit the benefits of object orientation. These include a higher level of abstraction, good reusability, high productivity, good maintainability, system integrity, and reliability. 
The proposed model is suitable for both simple and complicated measurement systems. In fact, it is capable of modeling networked or distributed measurement systems. As indicated in Fig. 3, the model generally supports multiple measuring instruments, multiple measured objects, or human observers.

With the encapsulation (or information hiding) and a higher level of abstraction, the model is closer to the nature view of the real-world and has highlighted the knowledge extraction of a measurement system. The proposed model is thus inherently consistent with the generalized concept of measurement, where the knowledge about the real-world is the ultimate purpose of any measurement.

The model reusability is a very important feature of an object-oriented modeling. Obviously, the potential reusability relies upon the availability of a library of previously developed objects or classes. Thus, the benefit may not be very clear at the early stage of the object-oriented modeling as only a small number of components have become available. Also difficulty may arises from the standardization for the use of these objects [7]. The specification of an object-oriented component is usually more complicated than functional procedures.

\section{Applications}

It should be pointed out that there is no unique implementation using an object-oriented approach. The actual model as an abstraction of a practical measurement system varies according to the purpose of the modeling and the understanding of system characteristics.

Serving as both a framework and a practical tool, the proposed model will certainly find applications in instrumentation engineering, and measurement research and education. As a framework, it has integrated various aspects of a measurement system. It also helps to identify and highlight the critical aspects of a measurement system.

As a practical tool, it can be used for the classification and systematic organization of measuring devices or systems, e.g., in the development of a relational database of measurement systems. The model also potentially allows for the evaluation of the performance of a measurement system. It will be generally useful in the description, analysis, and design of a measuring instrument or system.

As object-oriented methods are closely related to the software development, the proposed model can be readily applied to virtual instrumentation, where its use may be further extended to the full life-cycle of a virtual system including system implementation and maintenance. The model will also be applicable in networked or distributed virtual measurement systems, e.g., through the Internet and the World Wide Web.

\section{CONCLUSIONS}

An object-oriented model for measurement systems has been discussed in connection with the generalized concept of measurement [1]. The proposed model has the advantages of an object-oriented method. In addition, in the modeling of measurement systems, it has captured the system charac- teristics in a wider view. Its inherent consistency with the knowledge-oriented measurement concept has the potential to solve the problems associated with traditional functionoriented models. It will provide a more satisfactory solution to the modeling of a measurement system.

With the model as both a conceptual framework and a practical tool, its applications include classification and systematic organization of measuring devices; description, analysis, design and performance evaluation of measurement systems; and virtual instrumentation.

\section{REFERENCES}

[1] Q. Yang and C. Butler, "On framework of measurement science," in Proc. XV IMEKO World Congr., Tampere, Finland, June 1997, vol. 5, pp. 101-106.

[2] F. Abdullah, L. Finkelstein, S. H. Khan, and W. J. Hill, "Modeling in measurement and instrumentation-An overview," Measurement, vol. 14, pp. 41-53, Sept. 1994.

[3] P. H. Sydenham, "Unsolved problems of measurement. An international study," in Proc. XIII IMEKO World Congr., Torino, Italy, Sept. 1994, pp. 827-831.

[4] P. K. Stein, "The unified approach to the engineering of measurement systems for test \& evaluation-A brief survey," in Proc. IEEE Instrumentation Measurement Tech. Conf., Brussels, Belgium, 1996, pp. $1-28$.

[5] K. Bjørn, Object-Oriented Programming with Simula. Reading, MA: Addison-Wesley, 1989.

[6] J. Rumbaugh, M. Blaha, W. Premerlani, F. Eddy and W. Lorensen, Object-Oriented Modeling and Design. Englewood Cliffs, NJ: Prentice-Hall, 1991.

[7] H. J. W. Spoelder, A. H. Ullings and G. C. A. Groen, "Virtual instrumentation: A survey of standards and their interrelation," in Proc. IEEE Instrumentation Measurement Tech. Conf., Ottawa, Ont., Canada, 1997, pp. 676-681, 1997.

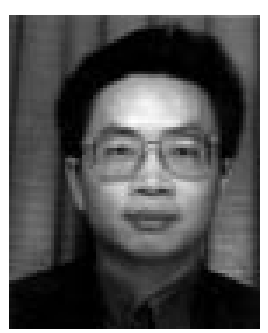

Qingping Yang (M'92) received the Diploma in instrumentation and measurement from Chengdu Aeronautical Polytechnic, Chengdu, China, in 1983. He was awarded a scholarship to study at Brunel University, Middlesex, U.K., in 1988, and received the Ph.D. degree in 1992.

He was an Assistant Engineer, Deputy Head of Department of Measurement and Testing in the Aircraft Structural Strength Research Institute (Ministry of Aerospace), Shaanxi Province, China. He currently lectures on instrumentation design, manufacturing metrology, and quality control. He has published more than 20 papers on sensors, transducers, instrumentation and measurement.

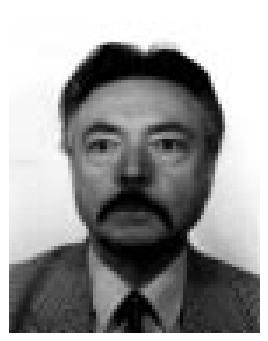

Clive Butler received the B.Sc degree in physics from the University of Manchester, Manchester, U.K., in 1963 and the M.Sc. and Ph.D. degrees from Imperial College, London, U.K., in 1968.

He has held a number of industrial posts including Chief Physicist, OMT Ltd., Director, Metronic Technology Ltd. and Digital Metrology Ltd. He was also Director of Research at Watford College of Technology, Watford, U.K. He is now Reader at Brunel University, Middlesex, U.K. His current research interests are in dimensional metrology and quality systems. He has presented more than 60 papers in applied optics, metrology, quality management, and printing technology.

Dr. Butler is a fellow of the Royal Society of Arts and member of the Institute of Quality Assurance. 